\title{
Nigeria's race to zero COVID-19 cases: True disease burden or testing failure?
}

\section{Ahmad Ibrahim Al-Mustapha ${ }^{1,2,3}$, Abubakar Ahmed Tijani ${ }^{4,5}$, Muftau Oyewo ${ }^{1,4}$, Ahmed Ibrahim $^{1}$, Nusirat Elelu ${ }^{6}$, Oluwaseun Adeolu Ogundijo ${ }^{2}$, Emmanuel Awosanya $^{2}$, Annamari Heikinheimo $^{7}$, Victoria Olusola Adetunji ${ }^{2}$}

\footnotetext{
${ }^{1}$ Department of Veterinary Services, Kwara State Ministry of Agriculture and Rural Development, Ilorin, Kwara State, Nigeria ${ }^{2}$ Department of Veterinary Public Health and Preventive Medicine, Faculty of Veterinary medicine, University of Ibadan, Oyo State, Nigeria

${ }^{3}$ Faculty of Pharmaceutical Sciences, Université de Tours, Tours, France

${ }^{4}$ Nigeria Field Epidemiology and Laboratory Training program, Asokoro, Abuja, Nigeria

${ }^{5}$ African Center for Disease Prevention and Control, Abuja, Nigeria

${ }^{6}$ Department of Veterinary Public Health and Preventive Medicine, Faculty of Veterinary Medicine, University of Ilorin, Kwara state, Nigeria

${ }^{7}$ Department of Food Safety and Environmental Health, Faculty of Veterinary Medicine, University of Helsinki, Finland
}

\section{T} he 2019 coronavirus disease (COVID-19), caused by a novel member of the beta coronaviruses, severe acute respiratory syndrome coronavirus 2 (SARS-CoV-2), has caused a global public health hazard [1]. Since Nigeria's index case on the 27 February 2020, the country has tested 1818957 persons, recorded 163195 confirmed cases and 2058 deaths as of 31 March 2021 [2]. The positivity rate among the tested population was $9.0 \%$ and the case fatality rate was 1.3\%. In April 2020, the Nigerian Government, through the Nigeria Center for Disease Control and Prevention (NCDC), activated its national Incident Control Center (ICC),

Nigeria's COVID-19 testing system is sub-optimal and this has contributed to the very low daily COVID-19 disease burden being reported. the Surveillance and Outbreak Response Management System (SORMAS), and the mobile strengthening epidemic response system (mSers) amongst others for national coordination, surveillance and reporting of COVID-19 cases in the country [3].

Nigeria's health care system is sub-optimal and plagued by a plethora of challenges as seen in many other low- and middle-income countries [4]. Nigeria's current health expenditure is 3.75\% of its gross domestic product (GDP) [5]. With a Physician to population ratio of 4:10000, Nigeria has one of the worst physicians to population ratio in the world [6]. The pandemic has further stretched the health care workforce, created panic in health facilities and made obvious the dilapidated situation of the country's health infrastructures $[3,4]$. Another major constraint to the success of Nigeria's COVID-19 control strategy was the high human poverty index (HPI). In Nigeria, an estimated 83 million people (40\%) live below the poverty line [7], thus making COVID-19 a disease of hunger [8]. 


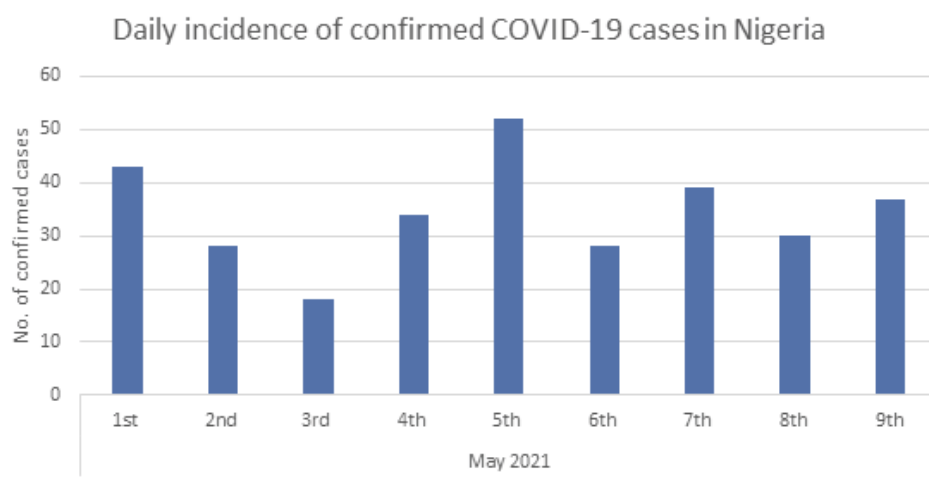

Figure 1. Daily incidence of confirmed COVID-19 cases in Nigeria as of 9 May 2021 [2].
An effective COVID-19 testing system is critical to understanding the pandemic's burden and spatio-temporal spread across the globe. This should enable the prompt identification of infected persons, determining populations most at risk, improve prognosis, and assist with contact tracing [3]. However, Nigeria's COVID-19 testing system and capacity are sub-optimal with close to only 2000000 people tested out of the estimated 210000000 population. Despite the low testing capacity, an even lower incidence of COVID-19 cases is being reported daily (Figure 1). Hence, this viewpoint asks the million-dollar question: Have we flattened the curve? And if yes, how soon do we get to zero cases? Furthermore, we evaluated Nigeria's COVID-19 testing capacity 14 months after the identification of patient zero.

\section{COVID-19 TESTING AND LABORATORY CAPACITY}

A reliable, fast, and efficient testing system is crucial to identifying cases and curbing the spread of COVID-19 especially in densely populated countries such as Nigeria (210 million people). Prior to the COVID-19 pandemic, Nigeria's laboratory networks and diagnostic capacities were poor. This was evident in the number of existing laboratories by January 2020, as only 6 BSL-3 laboratories with capacity to test for SARS-CoV2 existed in Nigeria. These facilities were mainly conducting quantitative polymerase chain reaction (RT-qPCR) and other confirmatory diagnostic tests for important endemic and sporadic outbreaks

The general public is advised to strictly adhere to COVID-19 preventive measures such as the use of masks, physical distancing, frequent washing of their hands. such as Lassa fever, Yellow fever and Cholera [3,4]. These laboratories soon became overwhelmed with the increasing COVID-19 incidence in Nigeria. As of April 2020, Nigeria could only test 2500 samples per day. To cater to the rising disease burden, NCDC leveraged on two existing platforms (HIV molecular testing laboratories as well as the GeneXpert M. tuberculosis testing equipment) and repurposed these machines to test for SARS-CoV-2. As of April 5, 2021, with increased commitment from the Government there now exists 124 COVID-19 testing centers in Nigeria. Of these, 76 are public owned COVID-19 testing facilities in the NCDC network, while 41 are fee-paying private COVID-19 testing laboratories and 7 corporate laboratories spread across Nigeria [2].

However, despite the expansion of the laboratory network across the country, only about 1.8 million samples have been tested across all platforms in Nigeria (open PCR, GeneXpert, and RDT kits) over 14 months (March 2020 - April 2021). This represents only $0.8 \%$ of Nigeria's population [9]. Table 1 puts into perspective, Nigeria's COVID-19 tests done in comparison with few selected countries. The average daily COVID-19 tests conducted rose from 2500 samples in April 2020 to 4000 samples by December 2020 and 5250 samples by April 2021. Currently, Nigeria has only tested 8.5 persons per thousand population. Hence, we opine that this extremely low testing system could be responsible for the low daily COVID-19 incidence in Nigeria.

Table 1. Cumulative COVID-19 tests conducted by selected countries as of 31 March 2021

\begin{tabular}{lcccc} 
Country & Population [10] & TOtal humans tested [9] & Tested PeR thousand [9] & Daily AVerage [9] \\
\hline Nigeria & 210134412 & 1803177 & 8.5 & 5250 \\
\hline Ethiopia & 117790090 & 2301145 & 19.8 & 8200 \\
\hline Morocco & 37245243 & 5443945 & 147.5 & 11300 \\
\hline South Africa & 59881455 & 9803871 & 165.3 & 27000 \\
\hline UAE & 9981853 & 37354626 & 3776.9 & 220000 \\
\hline United Kingdom & 68155831 & 124147198 & 1783.5 & 1216000 \\
\hline
\end{tabular}

\section{RAPID DIAGNOSTIC TESTS}

Although RT-PCR is the gold standard for diagnosing COVID-19, antigen-based rapid diagnostic tests (RDTs) was granted emergency use authorization by the World Health Organization (WHO) in September 2020 [11]. RDTs are cheap, fast, require less technical expertise and could be more sustainable in LMICs such 


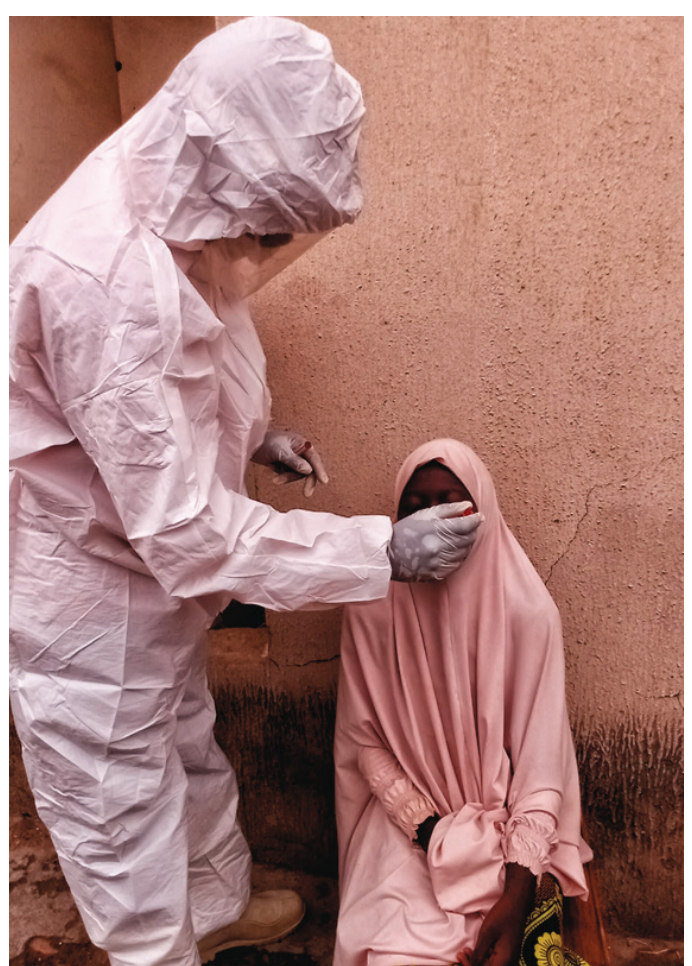

Photo: From the collection of Al-Mustapha Ahmad Ibrahim (used with permission). as Nigeria. Consequently, Nigeria validated it during the screening of youth corps before their admission into the compulsory one-year national service [12]. Ige et al. reported that although COVID-19 RDTs have lower sensitivity and specificity, they have proven useful in scaling up Nigeria's COVID-19 testing system especially in schools, congregations, and hard-to-reach areas [13]. However, it is yet to be determined if the low sensitivity of RDTs contributed to the lower COVID-19 incidence in Nigeria. COVID-19 testing using RDTs should be conducted by qualified health care personnel while adhering strictly to COVID-19 protocols to avoid technical errors and further community spread of the SARS-CoV-2 [13].

\section{THE NEED TO REVIEW THE NATIONAL COVID-19 TESTING PLAN}

To successfully navigate the nation out of this pandemic, the true burden of the COVID-19 pandemic must be determined especially in the race to zero cases. This can be achieved by increasing Nigeria's COVID-19 laboratory testing capacity across the nation. The current testing framework and strategies are ineffective and must be improved so that increased testing can be done to capture at least $5 \%$ of Nigeria's population by December 2021. Furthermore, we propose "risk-adjusted mass screening" of Nigerians. This is in line with the target of the Africa Center for Disease Control and prevention (Africa CDC) initiative "partnership to accelerate COVID-19 testing" (PACT) which aims to conduct at least 80000 tests per million population [14]. Some African countries have used this testing system to determine the spatio-temporal spread and the true burden of COVID-19 in their countries. Countries such as South Africa (9803871), Morocco (5443945) etc. have started the risk-based mass screening of their citizens [14]. In some of these countries, the local production of their nucleic acid extraction kits and laboratory reagents has assisted them in ramping up the number of tests conducted. PACT, Coalition against COVID-19 (CA-COVID), and other corporate organizations have assisted the Presidential Task Force (PTF) to increase testing, contact tracing, and treatment of all cases, which are cardinal components in reaching zero cases [14].

The need for community engagement towards any successful public health intervention cannot be overemphasized. Hence, to fully curtail the spread of the SARS-CoV-2, there is the need to employ community engagement strategies that will encourage adoption of all recommended safety measures through rigorous public education and community enforcement in order to prevent further spread of the virus within the country. This should be combined with rigorous contact tracing, improved testing strategy and mass vaccination with effective vaccines. The government must improve public trust in its handling of the pandemic through mass advocacy and public health education. Public trust in government is a crucial factor in vaccine acceptance, a cardinal instrument in curbing the spread of the SARS-CoV-2 and returning to normalcy. Therefore, it remains paramount for the government to sensitize and persuade its citizenry to "take responsibility" by strictly adhering to the infection prevention and control measures (use of face mask, frequent hand washing, physical distancing) and accepting the COVID-19 vaccine.

\section{CONCLUSION}

Although government's preparedness and response efforts towards the pandemic have been above average, more needs to be done especially in the area of testing. Nigeria has neither flattened the curve nor heading towards zero COVID-19 cases. The low daily disease incidence is mostly a function of a very low testing rate. There is a need to quickly roll out COVID-19 vaccination to cover $70 \%$ of the population so as to achieve herd immunity. Furthermore, a transparent, holistic, and participatory approach by the government is critical to combating the COVID-19 pandemic in Nigeria and rebuilding stronger health systems towards adjusting to a "new normal". 
Funding: This research did not receive any specific grant from funding agencies in the public, commercial, or not-forprofit sectors.

Authorship contributions: Authors contributed equally to this viewpoint. All authors reviewed and approved the final version for submission.

Competing interests: The authors completed the ICMJE Disclosure of Interest Form (available upon request from the corresponding author), and declare that they have no competing interests.

1 World Health Organization. Naming the coronavirus disease (COVID-19) and the virus that causes it. Who.int. 2021. Available: https://www.who.int/emergencies/diseases/novel-coronavirus-2019/technical-guidance/naming-the-coronavirus-disease(covid-2019)-and-the-virus-that-causes-it\#: :text=ICTV\%20announced\%20\%E2\%80\%9Csevere\%20acute,on\%2011\%20February\%202020. Accessed: 2 April 2021.

2 NCDC. Coronavirus Microsite. Covid19.ncdc.gov.ng. 2021. Available: https://covid19.ncdc.gov.ng/\#! Assessed: 1 April 2021.

3 Adesanya OA. Government preparedness and response towards COVID-19 outbreak in Nigeria: A retrospective analysis of the last 6 months. 2021. J Glob Health. 2020;10:020382. Medline:33214891 doi:10.7189/jogh.10.020382

4 Anthonia Obi-Ani N, Ezeaku D, Ikem O, Isiani M, Obi-Ani P, Janefrances Chisolum O. Covid-19 pandemic and The Nigerian primary healthcare system: The leadership question. Cogent Arts Humanit. 2021;8:1859075. doi:10.1080/23311983.2 020.1859075

5 World Bank. 2020b. Current health expenditure (\% of GDP) - Nigeria. 2020. Available: https://data.worldbank.org/indicator/ SH.XPD.CHEX.GD.ZS?locations=NG. Assessed: 1 April 2021.

6 World Bank. 2020a.Physicians (per 1,000 people) - Nigeria. Data. 2020. Available: https://data.worldbank.org/indicator/ SH.MED.PHYS.ZS?locations=NGWB GDP. Assessed: 1 April 2021.

7 World Bank. Nigeria releases new report on poverty and inequality in country | Living Standards Measurement Study (LSMS). 2020. Available: http://surveys.worldbank.org/blogs/nigeria-releases-new-report-poverty-and-inequality-country\#: :text=The\%20National\%20Bureau\%20of\%20Statistics,naira\%20(\%24381.75)\%20per\%20year. Assessed: 1 April 2021.

8 Kalu B. COVID-19 in Nigeria: a disease of hunger. Lancet Respir Med. 2020;8:556-7. Medline:32359414 doi:10.1016/S22132600(20)30220-4

9 Humanitarian Data Exchange. Total COVID-19 Tests Performed by Country. 2021. Available: https://data.humdata.org/m/ dataset/total-covid-19-tests-performed-by-country. Assessed: 1 April 2021.

10 Population > World - Worldometer. 2021. Available: https://www.worldometers.info/population/world/. Assessed: 1 April 2021.

11 World Health Organization. 19th WHO regulatory update on COVID-19. 2020. Available: http://www.who.int/docs. Assessed: 1 April 2021.

12 Guardian Newspaper. 2021. Available: https://t.guardian.ng/news/ncdc-okays-covid-19-rapid-test-kits-unveils-new-guideline/. Assessed: 1 April 2021.

13 Ige O, Buru A, Lamido T, Mohammed T, Dogara L, Patience I, et al. Evaluation of SARS-CoV2 antibody Rapid Diagnostic Test kits (RDTs) and Real Time-Polymerase Chain Reaction (Rt-PCR) for COVID-19 Diagnosis in Kaduna, Nigeria. 2020. MedRxiv Pre-print. doi:10.1101/2020.11.24.20231324

14 Africa CDC. COVID-19 Daily Updates. Africa CDC. 2020. Available: https://africacdc.org/covid-19/. Assessed: 1 April 2021.

\section{Correspondence to:}

Ahmad Ibrahim Al-Mustapha Department of Veterinary Public Health and Preventive Medicine Faculty of Veterinary Medicine, University of Ibadan, Oyo State Nigeria

ai.almustapha42@gmail.com 